\title{
Preparation of stable multiple emulsions using food-grade emulsifiers: evaluating the effects of emulsifier concentration, W/O phase ratio, and emulsification process
}

\author{
H. Ghasemi ${ }^{1} \cdot$ S. Darjani ${ }^{2} \cdot$ H. Mazloomi ${ }^{3} \cdot$ S. Mozaffari ${ }^{4}$
}

Received: 22 April 2020 / Accepted: 11 November 2020 / Published online: 16 November 2020

(c) Springer Nature Switzerland AG 2020

\begin{abstract}
In this study, the effects of sodium caseinate ( $\mathrm{NaCN}$; emulsifier) concentration, a type of hydrophilic emulsifier, as well as concentration of primary W/O emulsion on the stability of water-oil-water (W/O/W) emulsions were investigated. Emulsions were made using two different emulsification techniques including ultrasonic liquid processing and high pressure homogenization (HPH). Microscopy images of W/O/W emulsions in combination with droplet size analysis and viscosity measurements showed that the sample with a higher percentage of primary W/O emulsion ( $50 \mathrm{wt} \% \mathrm{vs.}$ $40 \mathrm{wt} \%$ and $25 \mathrm{wt} \%$ ) was more resistant to coalescence (narrower droplet size distribution and lower creaming index). The higher stability of this emulsion at $50 \mathrm{wt} \%$ is due to the enhancement in the solution viscosity which slows down the coalescence and destabilization kinetics. The increase in the $\mathrm{NaCN}$ concentration from $0.3 \mathrm{wt} \%$ to $0.9 \mathrm{wt} \%$ (based on total weight of emulsion) led to formation of larger droplets possibly due to the destabilization of primary W/O emulsion through the disruption of polyglycerol polyricinoleate (PGPR) layer. Regarding the effect of emulsifier type, incorporation of Cremophor EL and Tween 60 in comparison with $\mathrm{NaCN}$ resulted in formation of smaller droplet size due to their enhanced surface activity at the interface. Finally, we found that using high pressure homogenizer (HPH) instead of ultrasonic processor was detrimental to the emulsion stability and size distribution. These findings further provide new pathways to design emulsion systems for food and drug delivery applications.
\end{abstract}

Keywords Emulsion $\cdot \mathrm{W} / \mathrm{O} / \mathrm{W} \cdot$ Sodium caseinate $\cdot$ Creaming index $\cdot$ Viscosity $\cdot$ Ostwald ripening

\section{Introduction}

An emulsion is a colloidal dispersion of two immiscible liquids, where one phase is dispersed in another [1]. In the liquid phase, the main types of simple emulsions include oilin-water (O/W) and water-in-oil (W/O) emulsions. Multiple emulsions are more complex systems, where the dispersed phase is by itself an emulsion. Multiple emulsions, in particular water-oil-water (W/O/W), have gained increasing interest due to their wide range of applications in controlled release of drugs, food formulation with reduced fat, encapsulation of active ingredients, and cosmetics $[2,3]$. W/O/W emulsions are often obtained by emulsification of W/O into the secondary aqueous phase [4]. The main methods of generating W/O/W emulsion are one step and two step emulsification approaches. In the case of one step emulsification, strong mechanical agitation is required to induce phase inversion. At the initial stage

$\triangle$ S. Darjani, sdarjani@ccny.cuny.edu; $\bowtie$ S. Mozaffari, saeedm6@vt.edu| ${ }^{1}$ Department of Materials Science \& Engineering, College of Engineering \& Applied Science, University of Wisconsin-Milwaukee, Milwaukee, USA. ${ }^{2}$ Benjamin Levich Institute and Department of Chemical Engineering, City College of the City University of New York, New York, USA. ${ }^{3}$ Department of Chemical Engineering, College of Engineering, University of Tehran, Tehran, Iran. ${ }^{4}$ Department of Chemical Engineering, Virginia Polytechnic Institute and State University, Blacksburg, USA. 
W/O emulsion is formed, but a portion of emulsion shows phase inversion leading to formation of W/O/W emulsion. On the other hand, two-step emulsification techniques consist of making primary W/O emulsion using low HLB (hydrophilic-lipophilic balance) emulsifier(s) that strongly favors W/O and dispersing it in a secondary aqueous solution containing hydrophilic emulsifier [5]. One of the critical elements that determines the efficacy of this technique is the intrinsic stability of the internal W/O emulsion. It is worth mentioning that the presence of hydrodynamic perturbations during the second stage of emulsification must not lead to any significant breakdown of the primary W/O emulsion in order to obtain stable W/O/W emulsions [6].

A variety of apparatuses are used to produce W/O/W emulsions such as: high pressure homogenizers $(\mathrm{HPH})$, blenders, microfluidizers, membranes, etc. These different apparatuses not only can induce the size reduction but also affect the emulsification capacity and adsorption of emulsifier at the interface $[7,8]$. High pressure homogenizers are effective tools to produce fine droplets (nanoemulsions), which may not be easily obtained via mixers or rotor-stator homogenizers [9]. The difference in the emulsion droplet size is directly associated to the energy density. The higher the energy input/density, the smaller the droplet size [9]. However, sometimes such high energy can result in emulsion destabilization. A W/O/W emulsion's stability against coalescence is mainly determined by compositional factors including the internal/external aqueous phase, hydrophobic/hydrophilic emulsifiers, and the type of oil phase $[10,11]$.

Internal aqueous phases often consist of molecules and compounds that are encapsulated for specific applications (for examples, drug, sugar, nutrients, and other active agents). External aqueous phases usually consist of emulsifiers (e.g. proteins, natural/synthetic active agents), hydrocolloids, and viscosity modifiers [12].

Emulsifiers play critical role in the stability of emulsions due to their tendency to be adsorbed at the oil-water interface. These surface-active agents are responsible for lowering the interfacial tension and facilitating the formation of emulsion droplets. The type of emulsifier can determine which phase is most likely to be the continuous phase and which one will be the dispersed phase. Therefore, using the wrong emulsifier could result in an inverted emulsion $[5,13]$. For example, polyglycerol polyricinoleate (PGPR) which has low HLB value of $1.5 \pm 0.5$ strongly favors the formation of $\mathrm{W} / \mathrm{O}$ emulsions and it is commonly used in formulation of W/O/W emulsions [5, 14]. While type of emulsifier is shown to be an important factor in the stabilization of W/O/W, the emulsifier concentration can also play a major role in the stability mechanism. Nollet et al. demonstrated that by increasing the concentration of PGPR in sunflower oil, the stability of W/O/W emulsion could be greatly improved leading to enhanced encapsulation of active ingredient (vitamin B12 in this case) [14].

Alternatively, sodium caseinate $(\mathrm{NaCN})$, a milk protein, can form an elastic layer around the oil droplets and favor the formation of $\mathrm{O} / \mathrm{W}$ and as a result it is commonly used in the outer water phase of W/O/W [15]. For example, Jahaniaval et al. evaluated the physicochemical and functional properties of soluble $\mathrm{NaCN}$ fractions as a function of both $\mathrm{pH}(\sim 3-8)$ and temperature $\left(\sim 50-100^{\circ} \mathrm{C}\right)$. The authors found that $\mathrm{NaCN}$ emulsification capacity is highly dependent on the $\mathrm{pH}$ and temperature of solution as these factors directly influence the $\mathrm{NaCN}$ concentration and confirmation around the oil droplets, and consequently the emulsion stability [16]. In addition to the effects of emulsifier type and concentration on the properties of emulsion, the weight ratio of $\mathrm{W} / \mathrm{O}$ can also alter the stability and creaming behavior of W/O/W through altering the rheology of media. In two separate studies looking at O/W and W/O/W emulsion systems using food-grade emulsifiers, it was highlighted that as the fraction of disperse phase increases the viscosity increases and as a result reduces the chance of coalescence $[17,18]$.

Furthermore, adsorption of fine particles including micro/nanoparticles at the interface is shown to affect W/O/W emulsion stability through altering the rheological properties of the system (e.g. elasticity and rigidity of interface) [19-21].

Despite many efforts to understand the stability of multiple emulsions, a study investigating the effects of altering emulsifier concentration and portion of primary $\mathrm{W} / \mathrm{O}$ emulsion on emulsion stability, in combination with these particular food-grade emulsifiers in the soybean oil phase, has not yet been conducted. This study shows for the first time that increase in droplet size is governed by different mechanisms as the concentration of W/O changes in the system. Additionally, this work illustrates that using even three different emulsifiers still yields a stable emulsion with similar droplet sizes and therefore indicates that our method/process can be generalized to other emulsion systems.

The significant impacts of processing techniques on droplet size distribution, creaming behavior, and system stabilization/destabilization, are also are lacking in the literature. To fill this gap, we investigated the effects of: (i) portions of primary W/O emulsion ( $25 \mathrm{wt} \%, 40 \mathrm{wt} \%$, $50 \mathrm{wt} \%$ ); (ii) the concentration of hydrophilic emulsifier; $\mathrm{NaCN}$, in the external aqueous phase $(0.3 \mathrm{wt} \%, 0.6 \mathrm{wt} \%$, $0.9 \mathrm{wt} \%$ ); (iii) the type of hydrophilic emulsifiers used; and (iv) the different processing techniques used (ultrasonic vs. high pressure homogenization) on the formation and stability of W/O/W emulsions. In this work, different methods for generation of multiple emulsions along with relevant characterization tools (such as microscopy, size analysis, 
rheometry, etc.) for monitoring the emulsion stability will be presented. Then, the emulsion stability analysis including droplet size measurements, creaming index, and viscosity measurements under different experimental conditions will be conducted and the results will be compared with the literature. The results of this study can aid with the design of multiple emulsions with desired droplet sizes and rheological properties which can be directly used in formulation of low-fat food and encapsulation of active ingredients.

\section{Materials and methods}

\subsection{Materials}

Different chemicals including $\mathrm{NaCN}$, Cremophor $\mathrm{EL}$, Tween 60, sodium chloride, soybean oil and (Hydroxypropyl)methyl cellulose (HPMC) were obtained from SigmaAldrich. Polyglycerol polyricinoleate (PGPR) was obtained from Palsgaard. All materials were used without further purification.

\subsection{Emulsion preparation}

W/O/W emulsions were prepared using a two-step process. The formation and stability of W/O/W emulsions under various experimental conditions were evaluated. The experimental parameters include: portions of primary W/O emulsion ( $25 \mathrm{wt} \%, 40 \mathrm{wt} \%, 50 \mathrm{wt} \%$ ), concentration of hydrophilic emulsifier; $\mathrm{NaCN}$, in the external aqueous phase $(0.3 \mathrm{wt} \%, 0.6 \mathrm{wt} \%, 0.9 \mathrm{wt} \%)$, type of hydrophilic emulsifier; and the emulsification process (ultrasonic vs. high pressure homogenizer). For example, in one of the formulations (i.e. $\mathrm{W} / \mathrm{O}$ concentration $=50 \mathrm{wt} \%$ and $\mathrm{W} / \mathrm{O}$ ratio $=20 / 60), 20 \mathrm{~g}$ water containing sodium chloride (40 mg) was dispersed in the oil phase consisting of soybean oil (58.3 g), PGPR (1.5 g) and HPMC ( $0.2 \mathrm{~g})$. The mixtures were homogenized at $700 \mathrm{~W}$ using a Vibra-cell Ultrasonic liquid processor (Sonics \& Materials, Inc) at $37^{\circ} \mathrm{C}$ for 30 min to obtain the primary W/O emulsions. The second step involved the dispersion of primary emulsions into the aqueous solution containing hydrophilic emulsifier (e.g. $\mathrm{NaCN}$, Tween 60, Cremophor EL) using the same homogenizer for the duration of $15 \mathrm{~min}$. The schematic of W/O/W emulsion preparation is illustrated in Fig. 1. To control the temperature and prevent dramatic increases in temperature during the emulsification, an ice-water bath was used. The total weight of W/O/W emulsion samples were kept constant at $160 \mathrm{~g}$.

Additionally, we compared the effect of different processing techniques on the emulsion stability. In the second approach, the primary W/O emulsion was made using IKA
T-10 homogenizer at 15,000 rpm for $3 \mathrm{~min}$. The resulting emulsion was further cooled in the room temperature and then homogenized using high pressure homogenizer $(\mathrm{HPH}$, IKA) at 1000 bars for 8 cycles. The same procedure was used to prepare the final W/O/W emulsions.

\subsection{Optical microscopy}

An optical microscope was used to observe the multiple emulsion samples and their stability against coalescence under different experimental conditions. A drop of emulsion sample was deposited on a glass slide and then enclosed with a coverslip. In few cases, the samples were slightly diluted with DI water to improve the imaging quality. The morphology of emulsion droplets was further monitored via light microscope (Olympus, USA). Water-soluble dye was added to the primary W/O emulsion to help with monitoring the encapsulation of inner water droplets during the second emulsification step (i.e. W/O/W).

\subsection{Determination of average droplet size of W/O/W emulsions}

The average droplet size and size distribution of W/O/W emulsions were measured using Malvern MasterSizer MSE (Malvern Instruments). The droplet size distribution was measured in W/O/W emulsion after different storage times at the room temperature. To ensure the homogeneity of the samples before taking the measurements, the emulsion samples were agitated gently. The measurements were conducted under continuous agitation while using refractive index of 1.48 for soybean oil with the obscuration range set in the range of $5 \%$ and $15 \%$. To ensure the accuracy of our results, each measurement was repeated three times and the results were compared with the microscopy images, accordingly. The surface weighted mean diameter $(D[2,3])$ was calculated based on the following equation [22]:

$D_{[3,2]}=\frac{\sum n_{j} d_{j}^{3}}{\sum n_{j} d_{j}^{2}}$

Here $d_{j}$ and $n_{j}$ are the diameter and number of droplets with size $d_{j}$, respectively. It should be noted that the estimation of average diameter based on $D[2,3]$ agrees more closely with the microscopy images than those obtained from $D[3,4]$ (i.e. volume weighted mean diameter) and as such $D[2,3]$ is reported. Additionally, the polydispersity of size distribution can be extracted as follows [17]:

Span $=\frac{d_{0.9}-d_{0.1}}{d_{0.5}}$ 
(a)

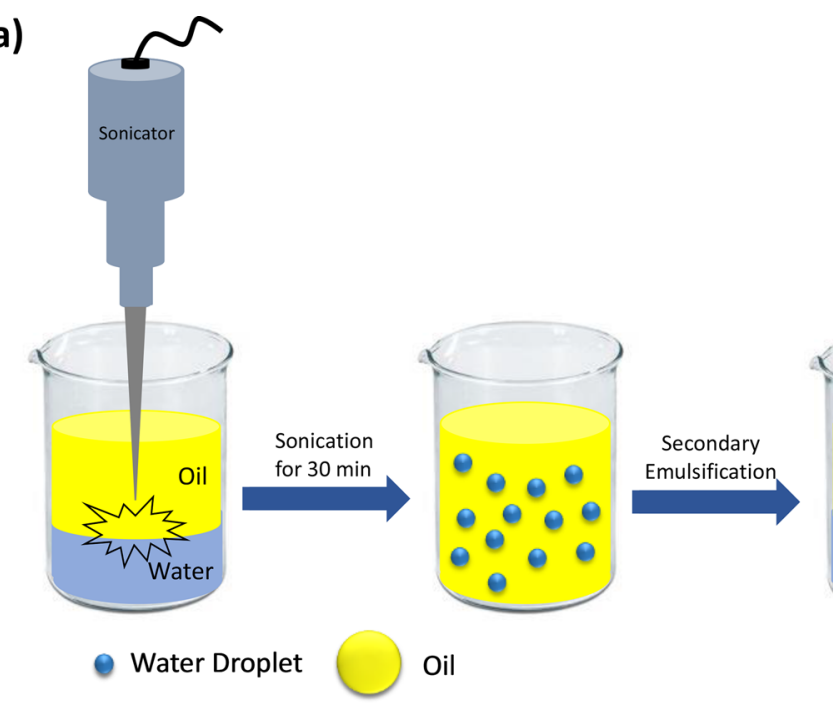

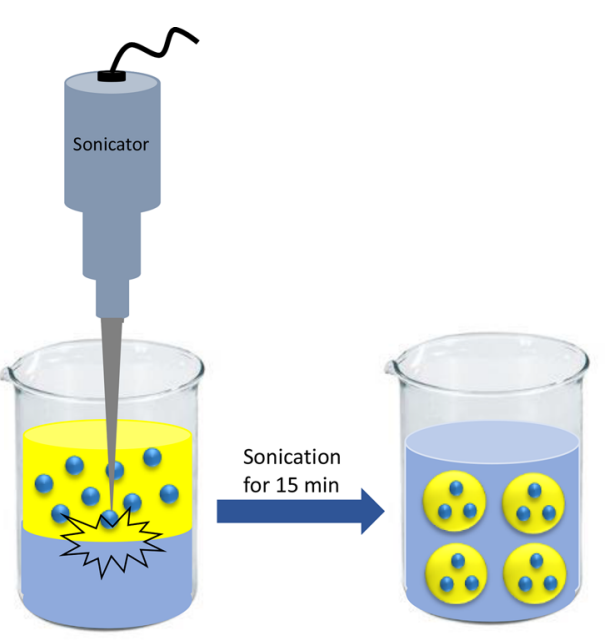

(b)

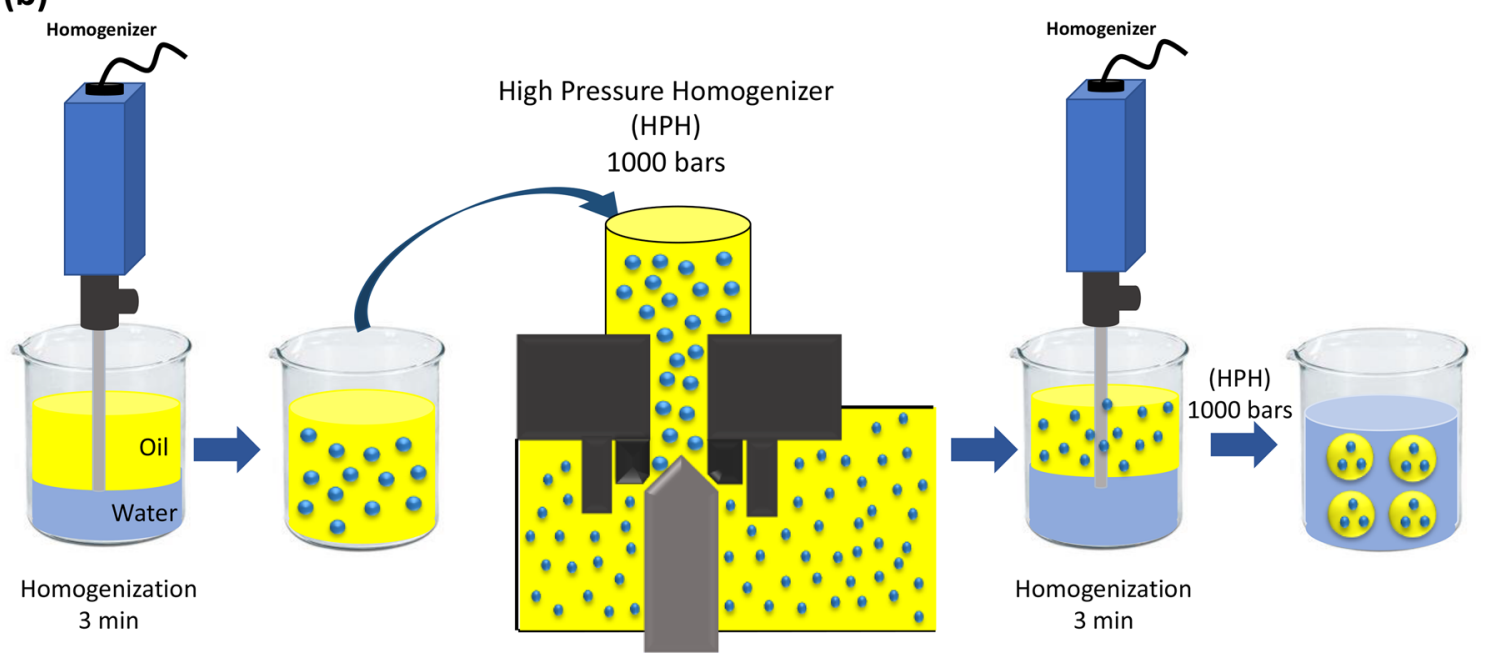

Fig. 1 Schematic of W/O/W emulsion preparation: $\mathbf{a}$ ultrasonic liquid processing and $\mathbf{b}$ high pressure homogenization (HPH)

where $d_{0.9}, d_{0.5}$, and $d_{0.1}$ account for the point in the size distribution in which $90 \%, 50 \%$, and $10 \%$ of the total volume of sample are contained.

\subsection{Stability tests for $\mathrm{W} / \mathrm{O} / \mathrm{W}$ emulsions as a function of storage time}

The stability of multiple emulsion samples was evaluated based on the observation of two separate phases: the cream layer and the serum layer at the top and bottom of the vials in ambient conditions, respectively. The creaming index (i.e. $\mathrm{H}_{L} / \mathrm{H}_{E} \%$; where $\mathrm{H}_{L}$ and $\mathrm{H}_{E}$ are the height of serum layer and total emulsion height, respectively) for different emulsion samples was calculated as a function of storage time. In this case, the higher creaming index value is indicative of emulsion with inferior stability.

\subsection{Viscosity measurements}

The rheological tests were conducted using an ARG2 rheometer (TA Instruments). Cone and plate geometry was used due to its compatibility with these emulsion systems. The viscosity of prepared emulsion samples was measured using cone contact angle of $2^{\circ}, 40 \mathrm{~mm}$ diameter, and with the $1000 \mu \mathrm{m}$ gap. The temperature was set to $23.00 \pm 0.15^{\circ} \mathrm{C}$ and the shear rate was varied between 0.2 and $150 \mathrm{~s}^{-1}$. For comparison purposes, the viscosity was reported at the shear rate of $15 \mathrm{~s}^{-1}$. For system calibration, water which has a known viscosity was used. The data was collected after the system reached equilibrium and the measurements were replicated three times to confirm the repeatability of our results. 


\section{Results and discussion}

\subsection{Effect of primary emulsion concentration on the formation and stability of W/O/W emulsions}

We evaluated the effect of altering the portion of primary W/O emulsion on the stability of multiple emulsions. Figure 2 shows the microscopy images of W/O/W emulsions prepared with the ultrasonic liquid processor along with their corresponding droplet size distribution. Based on the results, it can be inferred that the emulsion with the highest portion of W/O (i.e. 50 wt\%, Fig. 2a) is more stable against coalescence with narrower droplet size distribution. On the other hand, further reduction in the percentage of primary emulsion led to formation of larger droplets with broader size distribution ( 25 and $40 \mathrm{wt} \% \mathrm{~W} / \mathrm{O}$, Fig. $2 b$ and $c$ ). The average droplet size increased from $4 \pm 0.6$ to $9 \pm 2.5 \mu \mathrm{m}$ with a decrease in the concentration of $\mathrm{W} / \mathrm{O}$ from $50 \mathrm{wt} \%$ to $25 \mathrm{wt} \%$. Additionally, the microscopy images of diluted emulsions depicted in Fig. 3a and $b$ clearly confirm the formation of multiple emulsions as indicated from the presence of an inner aqueous phase. It is also shown that the oil phase can contain one and/or multiple water droplets depending on the emulsion type. These results agree well with the literature, showing the formation of multiple emulsions using microscopy techniques $[23,24]$. We note that the higher stability at the higher concentration (50 wt $\%$ ) of primary emulsion can
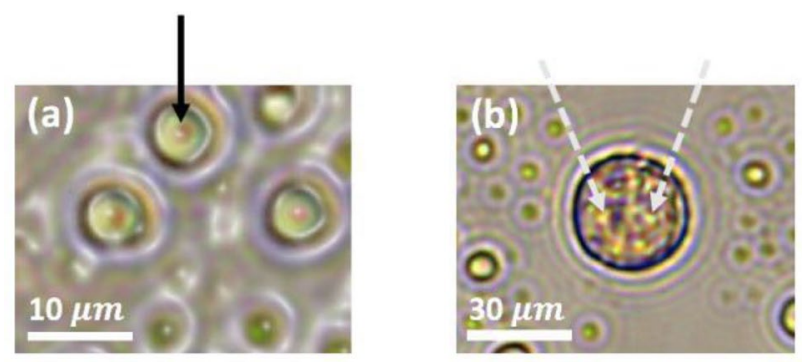

Fig. 3 Microscopy images of diluted W/O/W emulsions $(\mathrm{NaCN}=0.3$ wt $\%$ ) with different portions of primary emulsion: a $\mathrm{W} / \mathrm{O}=50 \mathrm{wt} \%$, b $\mathrm{W} / \mathrm{O}=40 \mathrm{wt} \%$. The arrows show the colored water droplets entrapped in the oil phase

be associated with increased solution viscosity due the larger volume fraction of dispersed phase [17]. To investigate how the rheology can play role in the stability of emulsions, the apparent viscosity of solutions at different portions of W/O were measured, accordingly. Based on the results shown in Fig. 4, it is clear that an increase in the portion of primary W/O emulsion, leads to an enhancement of solution viscosity. For example, the viscosity increases from $1.4 \pm 0.19$ to $4.7 \pm 0.21 \mathrm{~Pa}$.s as the W/O concentration altered from $25 \mathrm{wt} \%$ to $50 \mathrm{wt} \%$. The increase in the emulsion viscosity reduces the chance of droplet-droplet collision and slows down the coalescence and destabilization kinetics. The enhanced emulsion stability with the increase in the solution viscosity has been reported for different
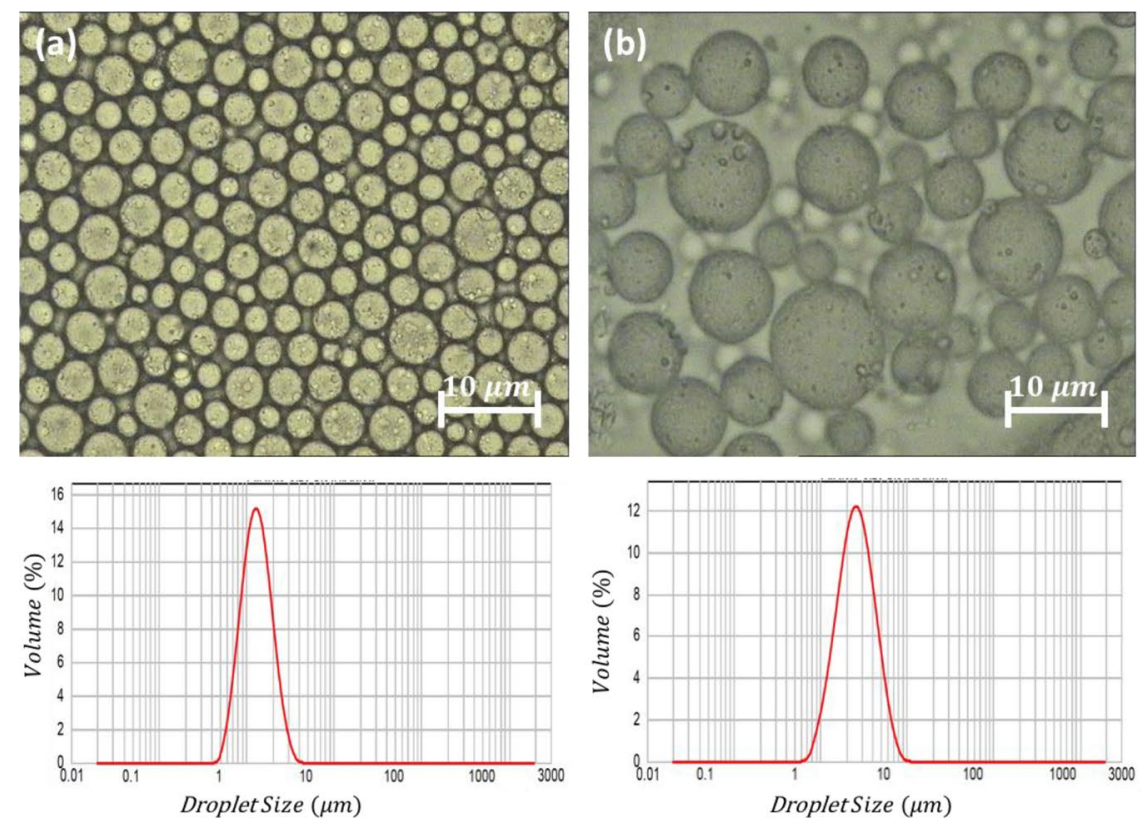

Fig. 2 Optical microscopy images and droplet size distribution of prepared W/O/W emulsions ( $\mathrm{NaCN}=0.3$ wt\%) with different portions of primary emulsion: a $\mathrm{W} / \mathrm{O}=50 \mathrm{wt} \%, \mathbf{b} \mathrm{W} / \mathrm{O}=40 \mathrm{wt} \%, \mathbf{c ~ W} / \mathrm{O}=25 \mathrm{wt} \%$ 


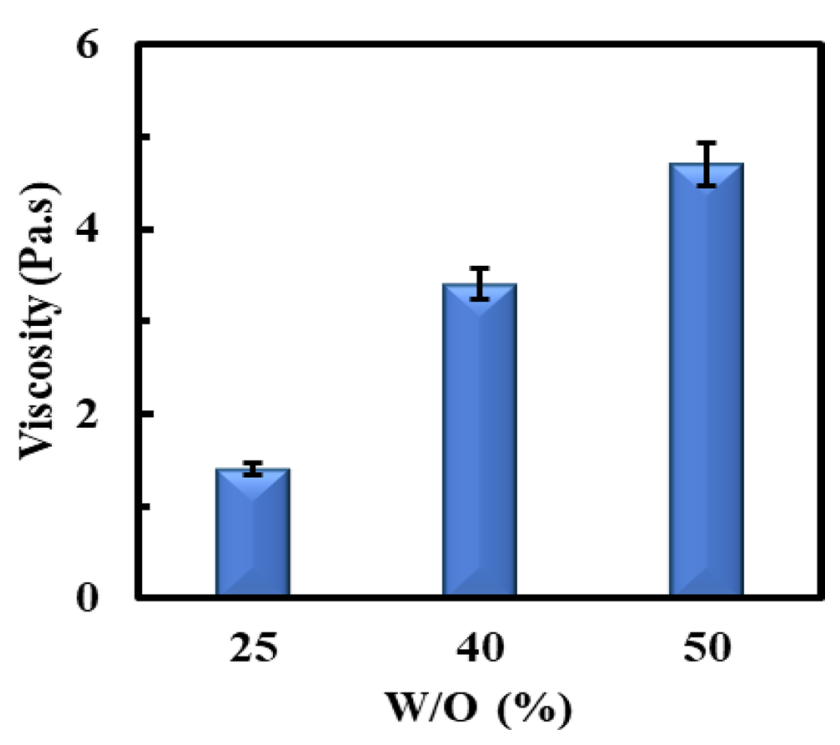

Fig. 4 Viscosity of W/O/W emulsions $(\mathrm{NaCN}=0.3 \mathrm{wt} \%)$ with different portions of primary emulsion $(25,40$, and $50 \mathrm{wt} \%)$ measured under ambient condition

multiple emulsion systems and support our results presented herein $[22,25]$.

Next, we calculated the creaming index $\left(\mathrm{H}_{L} / \mathrm{H}_{E} \%\right.$; where $\mathrm{H}_{\mathrm{L}}$ and $\mathrm{H}_{\mathrm{E}}$ are the height of serum layer and total emulsion height, respectively) of these emulsions at different storage times under ambient conditions. The results summarized in Fig. 5 show that the emulsion with the lower percentage of primary W/O emulsion possesses higher creaming index ( $21 \%$ vs. $12 \%$ for $25 \mathrm{wt} \%$ and $50 \mathrm{wt} \%$

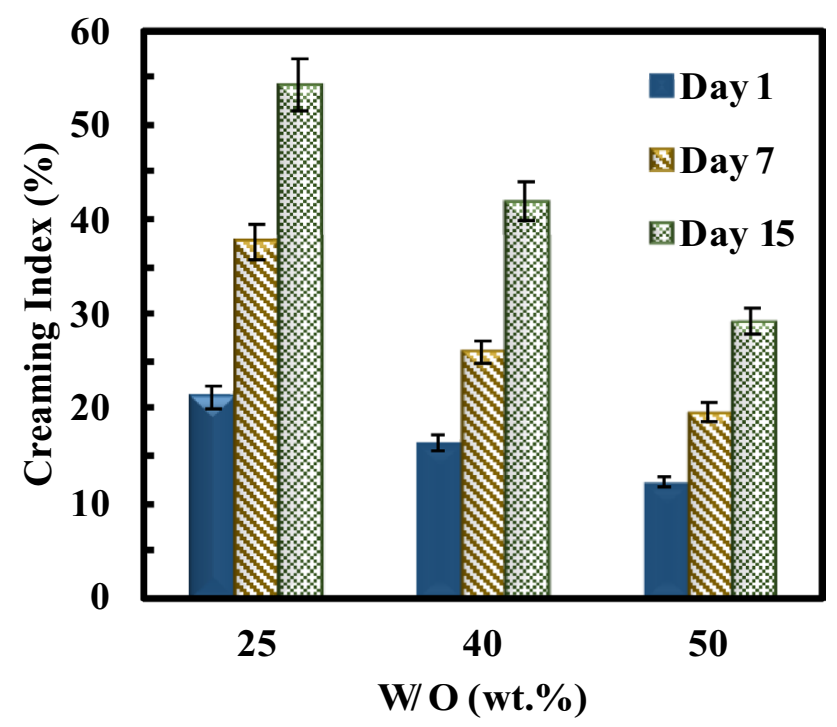

Fig. 5 Creaming Index (\%) at different storage times while using different portions of primary W/O emulsion ( $\mathrm{NaCN}=0.3 \mathrm{wt} \%): 25$ wt $\%, 40$ wt $\%$, and 50 wt $\%$
W/O after 1-day storage, respectively) with inferior stability than those with higher W/O percentage. The creaming index results agree well with the microscopy images and the droplet size measurements shown in Figs. 2 and 3. The increase of water phase can also induce greater instability, possibly due to the increased frequency of coalescence and bridging at higher water volume fraction. The increase in the creaming index and further emulsion destabilization at the longer storage times originate from higher rates of flocculation and coalescence [26].

To further evaluate the possible mechanism behind the increase in the droplet size, we plotted $\mathrm{D}^{3}$ ave as a function of time ( $\mathrm{D}^{3}$ ave vs. $\mathrm{t}$ ) for the samples with different portions of primary W/O emulsion (see Fig. 6). The linear correlation between $\mathrm{D}^{3}{ }_{\text {ave }}$ vs. $t$ for the case of $25 \mathrm{wt} \% \mathrm{~W} / \mathrm{O}$ further indicates that the increase in droplet size is mainly governed by Ostwald ripening [27]. Nevertheless, at higher portions of W/O (i.e. $40 \mathrm{wt} \%$ and $50 \mathrm{wt} \%$ ) $\mathrm{D}_{\text {ave }}^{3}$ showed insignificant dependency to $t$ (storage time) and therefore such a small increase in the size can originate from simple droplet-droplet interactions and further droplets coalescence. We also evaluated the effects of $\mathrm{NaCN}$ concentration on the droplet size distribution and emulsion stability against creaming to better understand how the concentration of emulsifiers alters the integrity of W/O/W emulsions.

\subsection{Effect of $\mathrm{NaCN}$ concentration on the formation and stability of W/O/W emulsions}

$\mathrm{NaCN}$ is shown to be a potential candidate for stabilization of W/O/W emulsions. $\mathrm{NaCN}$ is often used as the hydrophilic emulsifier and can impact the stability of W/O/W emulsion in the presence of a hydrophobic emulsifier such as PGPR [28]. Based on the concentrations of $\mathrm{NaCN}$ and PGPR in the emulsion the coverage of droplets with emulsifiers and droplet-droplet interactions can be easily influenced

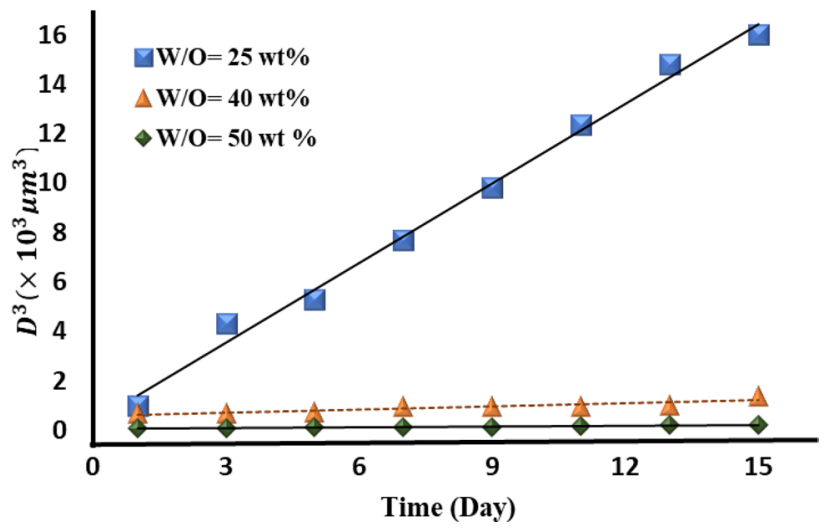

Fig. $6 D^{3}$ ave as a function of time $\left(D^{3}\right.$ ave vs. t) for the samples with different portions of primary W/O emulsion ( $\mathrm{NaCN}=0.3 \mathrm{wt} \%)$ : 25 wt $\%, 40$ wt $\%$, and 50 wt $\%$ 
resulting in formation of emulsions with different size distribution and creaming stability [29].

We monitored the stability of W/O/W emulsion as a function of $\mathrm{NaCN}$ concentration, while keeping other conditions the same $(\mathrm{W} / \mathrm{O}=50 \mathrm{wt} \%)$. Figure 7 reveals that with increase in the concentration of $\mathrm{NaCN}$ in the external aqueous phase from $0.3 \mathrm{wt} \%$ to $0.9 \mathrm{wt} \%$, the average droplet size slightly increases, and size distribution becomes broader. This trend is consistent with the higher creaming index values observed at the higher $\mathrm{NaCN}$ concentration (see Fig. 8). It should be noted that this result may seem counter-intuitive, as increasing $\mathrm{NaCN}$ concentration in the solution should lead to higher coverage of oil droplets and therefore favor the formation of smaller droplets [28].

Nevertheless, we believe that formation of larger droplets in our emulsion system can be attributed to the disruption of the PGPR layer with increase in $\mathrm{NaCN}$ concentration at the W/O interface (since $\mathrm{NaCN}$ can diffuse in and out of the external aqueous phase). In the next section we aim to gain a deeper understanding of system stability, and evaluate the effects of emulsifier type as well as processing techniques (ultrasonic liquid processing vs. high pressure homogenization) on droplet size and size distribution in W/O/W emulsions.

\subsection{Effect of hydrophilic emulsifier type and processing techniques on the droplet size distribution of W/O/W emulsions}

Emulsifier type can play significant role in altering the properties of emulsions. Milk protein (e.g. $\mathrm{NaCN}$ studied herein) can adsorb and unfold at the oil-water interface and increase the droplet surface coverage [30]. The correct selection of emulsifier results in the formation of a stable emulsion with desired physicochemical and functional properties. For example, Zamani et al. showed that replacing Tween 20 with whey protein microgels (WPMs) in the emulsion can significantly improve the stability and prevent phase separation due to the enhancement

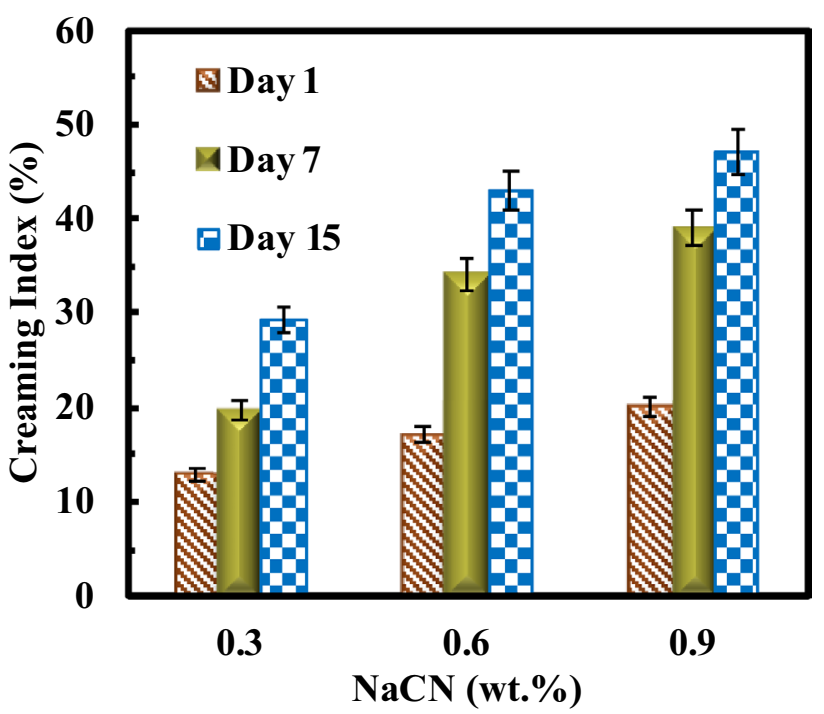

Fig. 8 Creaming Index (\%) at the different storage times for different concentrations of $\mathrm{NaCN}$ in the external aqueous phase: 0.3 wt $\%, 0.6 \mathrm{wt} \%$ and $0.9 \mathrm{wt} \%$

of viscoelastic properties of emulsion (elastic modulus and yield stress) [19].

Based off the findings of Zamani et al. we replaced the $\mathrm{NaCN}$ with other types of emulsifiers including Tween 60 and Cremophor EL (W/O = 50 wt\%, emulsifier concentration $=0.3 \mathrm{wt} \%$ ). The droplet size analysis along with creaming behavior analysis showed small differences between these different cases. The two emulsion samples containing Tween 60 and Cremophor EL vs. the sample of $\mathrm{NaCN}$ showed slightly smaller droplet size with narrower size distribution possibly due to their enhanced surface activity at the interface (see Fig. 9). For example, the average droplet sizes for $\mathrm{NaCN}$, Tween 60 , and Cremophor EL were $4 \pm 0.6 \mu \mathrm{m}, 3.1 \pm 0.5 \mu \mathrm{m}$, and $2.4 \pm 0.4 \mu \mathrm{m}$, respectively. This slight dependency of emulsion stability and droplet size to the types of hydrophilic emulsifier could be due to: (i) greater stability of
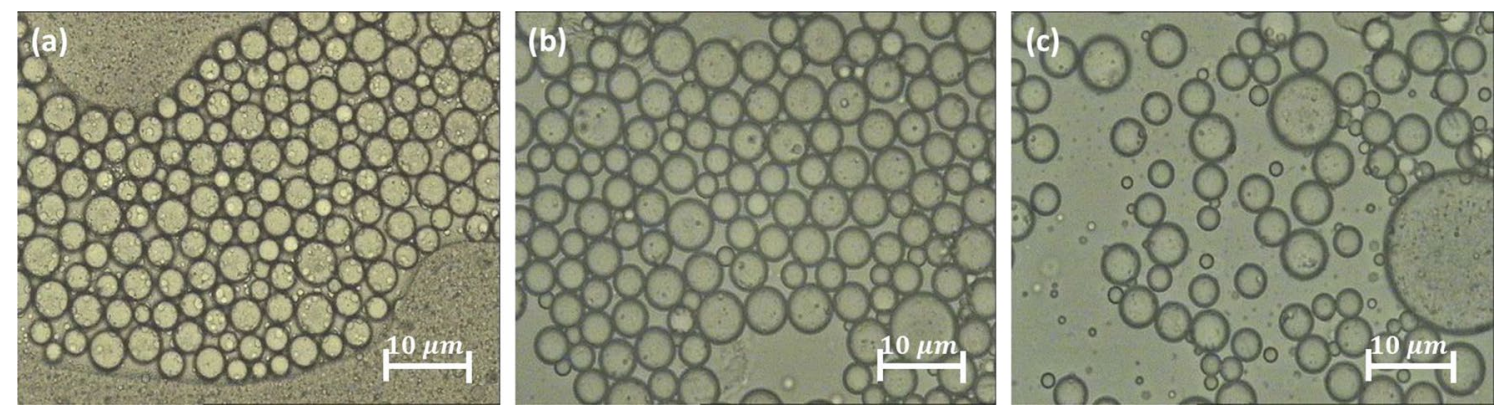

Fig. 7 Microscopy images of W/O/W emulsions with different concentrations of $\mathrm{NaCN}$ in the external aqueous phase $(\mathrm{W} / \mathrm{O}=50 \mathrm{wt} \%)$ : a $\mathrm{NaCN}=0.3 \mathrm{wt} \%$ b $\mathrm{NaCN}=0.6 \mathrm{wt} \%$ and $\mathbf{c} \mathrm{NaCN}=0.9$ wt $\%$ 


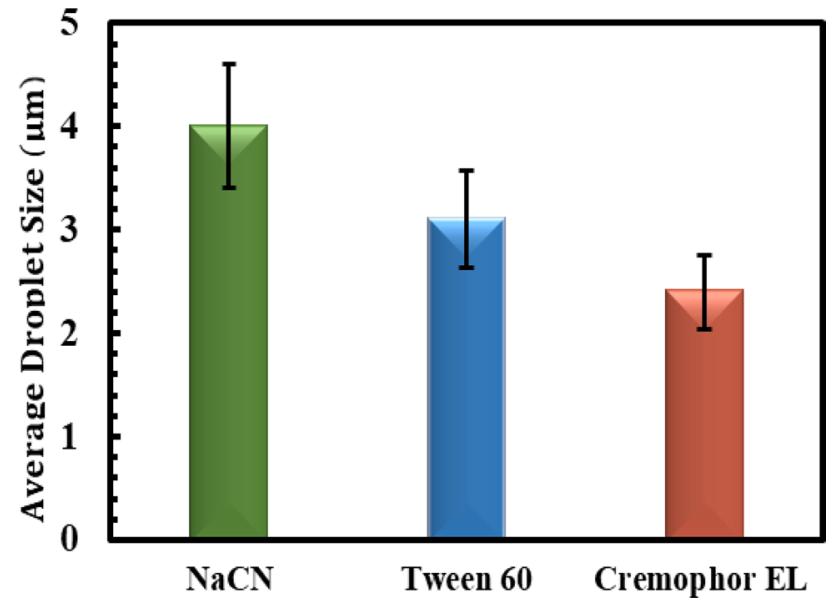

Fig. 9 Average droplet size of W/O/W emulsions while using different hydrophilic emulsifiers including: $\mathrm{NaCN}$, Tween 60 and Cremophor EL (W/O = $50 \mathrm{wt} \%$ and emulsifier concentration $=0.3 \mathrm{wt} \%)$

the primary emulsion where PGPR prevents coalescence due to its high droplet surface coverage; and/or (ii) the synergetic effect of hydrophilic emulsifier with the hydrophobic one at this specific experimental condition (e.g. concentration, temperature, and etc.). However, in our study, although the type of emulsifier did not influence the emulsion stability to a great extent, but it may still be important for other emulsions systems including high internal phase emulsions (HIPE) and O/W/O [31, 32].

Finally, we evaluated the effect of processing techniques (ultrasonic liquid processing vs. high pressure homogenization) on the emulsion stability against creaming and examined the droplet size distribution while using $\mathrm{NaCN}$ as the emulsifier. As shown in Fig. 10, the use of $\mathrm{HPH}$ instead of the ultrasonic liquid processor adversely affected the emulsion stability and droplet size distribution. The emulsion prepared with HPH showed fast creaming and significant phase separation even during one day of storage time. These results are not surprising and worth discussing in more detail. Since HPH is a high-energy emulsification method, it can destabilize the primary W/O and/or the whole W/OW emulsions given these systems are very sensitive to the energy input $[3,33]$.

We believe this is also the main reason why HPH in comparison with the ultrasonication method leads to the formation of emulsions with inferior stability and significantly broader size distribution (see Fig. 10a and b). Another possible scenario could be the adverse effects of $\mathrm{HPH}$ on the properties of ingredients (e.g. emulsifier) present in the emulsions. For example, HPH can affect the adsorption kinetics of emulsifiers at the interface and alter the rheological properties of multiple emulsion. (a) Ultrasonic liquid Processor

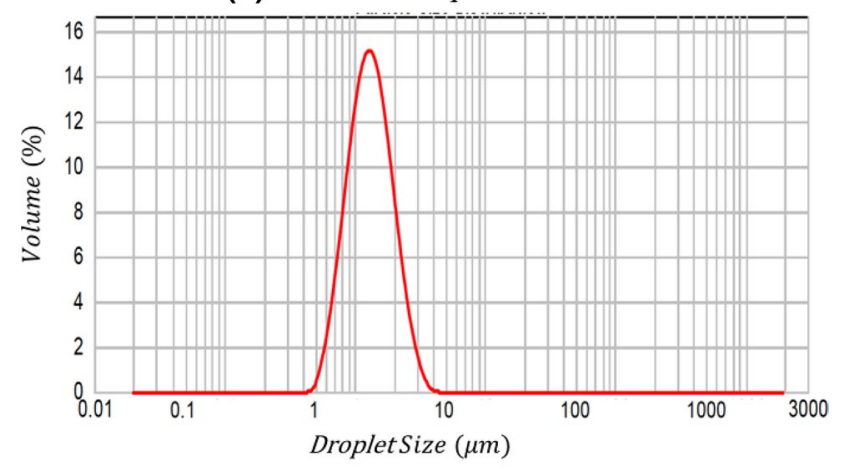

(b) High Pressure Homogenizer (HPH)

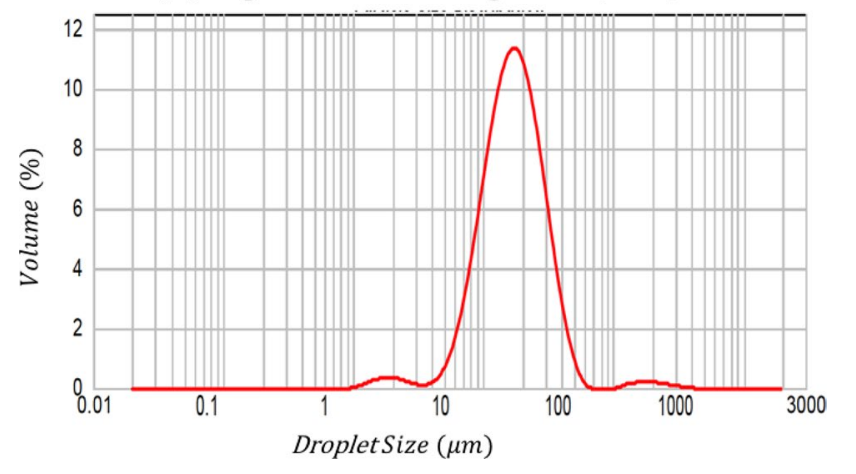

Fig. 10 Droplet size distribution of W/O/W emulsions prepared via using different processing techniques: a ultrasonic liquid processor and $\mathbf{b}$ high pressure homogenizer $(\mathrm{HPH})$. Experimental conditions: $\mathrm{W} / \mathrm{O}=50 \mathrm{wt} \%$ and $\mathrm{NaCN}=0.3 \mathrm{wt} \%$

\section{Conclusion}

In this research study, we examined the effects of sodium caseinate $(\mathrm{NaCN})$ concentration, a type of hydrophilic emulsifier, portions of primary W/O emulsion, and the emulsification process on the formation and stability of W/O/W emulsions. The analysis of W/O/W emulsions revealed that the sample with higher concentration of primary W/O emulsion (50 wt $\%$ vs. $40 \mathrm{wt} \%$ and $25 \mathrm{wt} \%$ ) possessed narrower droplet size distribution and a lower creaming index. In this case, the average droplet size decreased from $9 \pm 2.5 \mu \mathrm{m}$ to $4 \pm 0.6 \mu \mathrm{m}$ as W/O concentration increased from $25 \mathrm{wt} \%$ to $50 \mathrm{wt} \%$. The higher stability of the emulsion at $\mathrm{W} / \mathrm{O}=50 \mathrm{wt} \%$ is attributed to the enhancement in the emulsion viscosity (larger volume fraction of dispersed phase) and slower rate of droplet coalescence. Increasing the $\mathrm{NaCN}$ concentration from 0.3 wt $\%$ to 0.9 wt $\%$ resulted in the formation of larger droplets possibly due to the destabilization of primary W/O emulsion through the disruption of the polyglycerol polyricinoleate (PGPR) layer by $\mathrm{NaCN}$ at the interface. Additionally, we evaluated the effects of different hydrophilic emulsifiers in the external aqueous phase and our results 
demonstrated that Cremophor EL and Tween 60 vs. $\mathrm{NaCN}$ lead to the formation of smaller droplet sizes, possibly due to their enhanced surface activity at the interface. Finally, the use of high pressure homogenization $(\mathrm{HPH})$ instead of ultrasonic liquid processing to generate $\mathrm{W} / \mathrm{O} / \mathrm{W}$ samples adversely affected the emulsion stability and led to formation of droplets with a broad size distribution. Our findings will help with the design of emulsion systems for desired applications using food-grade emulsifiers.

Acknowledgements The authors are grateful to the Department of Chemical Engineering at University of Tehran and Materials Research Institute (MRI) for providing us with the financial support and allowing us to use their equipment. We are also very grateful to Mr. Hajipour for fruitful discussions regarding the possible effects of emulsification process on the formation and stability of multiple emulsions. Finally, we express our appreciation to Dr. Pishvai for his invaluable feedback.

\section{Compliance with ethical standards}

Conflict of interest The authors have no conflict of interest to report.

\section{References}

1. McClements DJ (2015) Food emulsions: principles, practices, and techniques. CRC Press, Boca Raton

2. Mataumoto S, Kang W (1989) Formation and applications of multiple emulsions. J Dispers Sci Technol 10(4-5):455-482

3. Muschiolik G, Dickinson E (2017) Double emulsions relevant to food systems: preparation, stability, and applications. Compr Rev Food Sci Food Saf 16(3):532-555

4. Sagalowicz L, Leser ME (2010) Delivery systems for liquid food products. Curr Opin Colloid Interface Sci 15(1-2):61-72

5. Benichou A, Aserin A, Garti N (2001) Polyols, high pressure, and refractive indices equalization for improved stability of W/O emulsions for food applications. J Dispers Sci Technol 22(2-3):269-280

6. van der Graaf S, Schroën C, Boom R (2005) Preparation of double emulsions by membrane emulsification-a review. J Membr Sci 251(1-2):7-15

7. Perrier-Cornet J, Marie P, Gervais P (2005) Comparison of emulsification efficiency of protein-stabilized oil-in-water emulsions using jet, high pressure and colloid mill homogenization. J Food Eng 66(2):211-217

8. Jaber WS, Alwared Al (2019) Removal of oil emulsion from aqueous solution by using Ricinus communis leaves as adsorbent. SN Appl Sci 1(8):944

9. Schubert $H$, Engel $R$ (2004) Product and formulation engineering of emulsions. Chem Eng Res Des 82(9):1137-1143

10. Taisne L, Walstra P, Cabane B (1996) Transfer of oil between emulsion droplets. J Colloid Interface Sci 184(2):378-390

11. Keshmiri K et al. (2016) Using microfluidic device to study rheological properties of heavy oil. arXiv preprint

12. Garti N, Aserin A (1996) Pharmaceutical emulsions, double emulsions, and microemulsions. Drugs Pharm Sci 73:411-534

13. Kanouni M, Rosano H, Naouli N (2002) Preparation of a stable double emulsion (W1/O/W2): role of the interfacial films on the stability of the system. Adv Coll Interface Sci 99(3):229-254
14. Nollet $\mathrm{M}$ et al (2018) Determination of formulation conditions allowing double emulsions stabilized by PGPR and sodium caseinate to be used as capsules. Langmuir 34(8):2823-2833

15. Ye A, Singh H (2001) Interfacial composition and stability of sodium caseinate emulsions as influenced by calcium ions. Food Hydrocoll 15(2):195-207

16. Jahaniaval $\mathrm{F}$ et al (2000) Soluble protein fractions from $\mathrm{pH}$ and heat treated sodium caseinate: physicochemical and functional properties. Food Res Int 33(8):637-647

17. Yildirim M, Sumnu G, Sahin S (2017) The effects of emulsifier type, phase ratio, and homogenization methods on stability of the double emulsion. J Dispers Sci Technol 38(6):807-814

18. Lu W, Zheng B, Miao S (2018) Improved emulsion stability and modified nutrient release by structuring $\mathrm{O} / \mathrm{W}$ emulsions using konjac glucomannan. Food Hydrocoll 81:120-128

19. Zamani $S$ et al (2018) Formation of shelf stable pickering high internal phase emulsions (HIPE) through the inclusion of whey protein microgels. Food Funct 9(2):982-990

20. Mozafari S (2015) Rheology of Bitumen at the onset of asphaltene aggregation and its efects on the stability of water-in-oil emulsion. Masters Thesis, University of Alberta, Canada

21. Ghasemi H et al (2020) High efficiency decolorization of wastewater by Fenton catalyst: magnetic iron-copper hybrid oxides. J Water Process Eng 37:101540

22. Matos M et al (2014) Preparation of water-in-oil-in-water (W1/O/ W2) double emulsions containing trans-resveratrol. Colloids Surf A 442:69-79

23. Khadem B, Sheibat-Othman N (2019) Theoretical and experimental investigations of double emulsion preparation by ultrasonication. Ind Eng Chem Res 58(19):8220-8230

24. Mahmood T et al (2013) Fabrication, physicochemical characterization and preliminary efficacy evaluation of a W/O/W multiple emulsion loaded with 5\% green tea extract. Braz J Pharm Sci 49(2):341-349

25. McClements D (2004) Principles, food emulsions: practice and techniques, 2nd edn. CRC Press, Boca Raton

26. Dapčević Hadnađev T et al (2013) Influence of oil phase concentration on droplet size distribution and stability of oil-in-water emulsions. Eur J Lipid Sci Technol 115(3):313-321

27. Wooster TJ, Golding M, Sanguansri $P$ (2008) Impact of oil type on nanoemulsion formation and Ostwald ripening stability. Langmuir 24(22):12758-12765

28. Su J et al (2006) Synergistic effects of polyglycerol ester of polyricinoleic acid and sodium caseinate on the stabilisation of water-oil-water emulsions. Food Hydrocoll 20(2-3):261-268

29. Chanamai R, McClements DJ (2000) Creaming stability of flocculated monodisperse oil-in-water emulsions. J Colloid Interface Sci 225(1):214-218

30. Euston SR, Hirst R (2000) The emulsifying properties of commercial milk protein products in simple oil-in-water emulsions and in a model food system. J Food Sci 65(6):934-940

31. Lei $L$ et al (2016) High internal phase emulsion with double emulsion morphology and their templated porous polymer systems. J Colloid Interface Sci 483:232-240

32. Thanh Diep T et al (2018) Double emulsion oil-in water-in oil (O/W/O) stabilized by sodium caseinate and k-carrageenan. J Dispers Sci Technol 39(12):1752-1757

33. Ding $S$ et al (2017) A new method for the formulation of double nanoemulsions. Soft Matter 13(8):1660-1669

Publisher's Note Springer Nature remains neutral with regard to jurisdictional claims in published maps and institutional affiliations. 